\title{
Continuous Roll-to-roll Production of Carbon Nanoparticles from Candle Soot
}

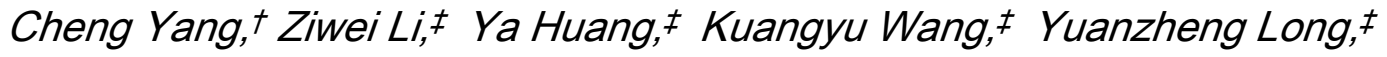 \\ Zeliang Guo, $\neq$ Xiaoyan $\mathrm{Li}^{*},{ }^{\dagger}$ and Hui Wu${ }^{*}, \neq$ \\ † Center for Advanced Mechanics and Materials Applied Mechanics \\ Laboratory Department of Engineering Mechanics Tsinghua University, \\ Beijing 100084, China \\ ‡ State Key Laboratory of New Ceramics and Fine Processing School of \\ Materials Science and Engineering, Tsinghua University, Beijing 100084, \\ China
}

\section{Corresponding Authors}

*E-mail: xiaoyanlithu@mail.tsinghua.edu.cn

*E-mail: huiwu@tsinghua.edu.cn 


\section{Supporting Information.}

\section{Experiment Section}

Chemicals and Materials

Polydimethylsiloxane (PDMS, base and curing agent, Sylgard 184, Dow Corning Co., LTD.), hexane (AR, Sinopharm Chemical Reagent Co., LTD.), stearic acid (98\%, Shanghai Aladdin Bio-Chem Technology Co., LTD.), ethanol (99.7\%, Modern Oriental (Beijing) Technology Development Co., LTD.), deionized water, matt black paint (purchased in local market), candle (purchased in local market)

\section{Preparation of CNPs}

The overall product yield was about $6.2 \%$.

Fabrication of anti-icing surface

PDMS (base $1 \mathrm{~g})$, curing agent $(0.1 \mathrm{~g})$, and hexane $(1.5 \mathrm{~g})$ were mixed with a mass ratio of 10:1:15. After 30 min stirring, the solution was sprayed on a substrate and the substrate was placed in a $65{ }^{\circ} \mathrm{C}$ drying oven for $18 \mathrm{~h} .0 .8 \mathrm{~g}$ carbon nanoparticles (CNPs) obtained from the bottom of the candle flame and $0.2 \mathrm{~g}$ stearic acid were dispersed into $100 \mathrm{~g}$ ethanol by ultrasonic dispersion instrument for $30 \mathrm{~min}$. The mixture was sprayed on to the substrate covered with PDMS under $80{ }^{\circ} \mathrm{C}$ heating. After cooling the CPS coating was obtained. A $0.1 \mathrm{~m}^{2}$ CNS coating could be produced in $10 \mathrm{~min}$ by spraying method. 
Frost melting and ice melting experiments

The temperature was controlled by a temperature chamber (Bluepard, BPHJ060A, China) and a cryogenic thermostat (YMNL-DC-0506, China). These experiments were achieved by a temperature control system. See also Figure S8.

Characterization

The structure of candle soot and CPS were characterized by SEM (FESEM, Leo-1530, Zeiss, Germany) and TEM (Jeol-2010, Japan). Raman spectra data were detected by laser Raman spectrometer (Evolution, HORIBA, France). Infrared spectroscopy information was obtained by FTIR spectrometer (TG-MSFTIR, Netzsch-X70, Germany). Composition information was detected by X-ray photoelectron spectrometry (Escalab 250 xi, Thermo Fisher). Infrared information and temperature were recorded by thermal imager (CEM-DT-980, China). 


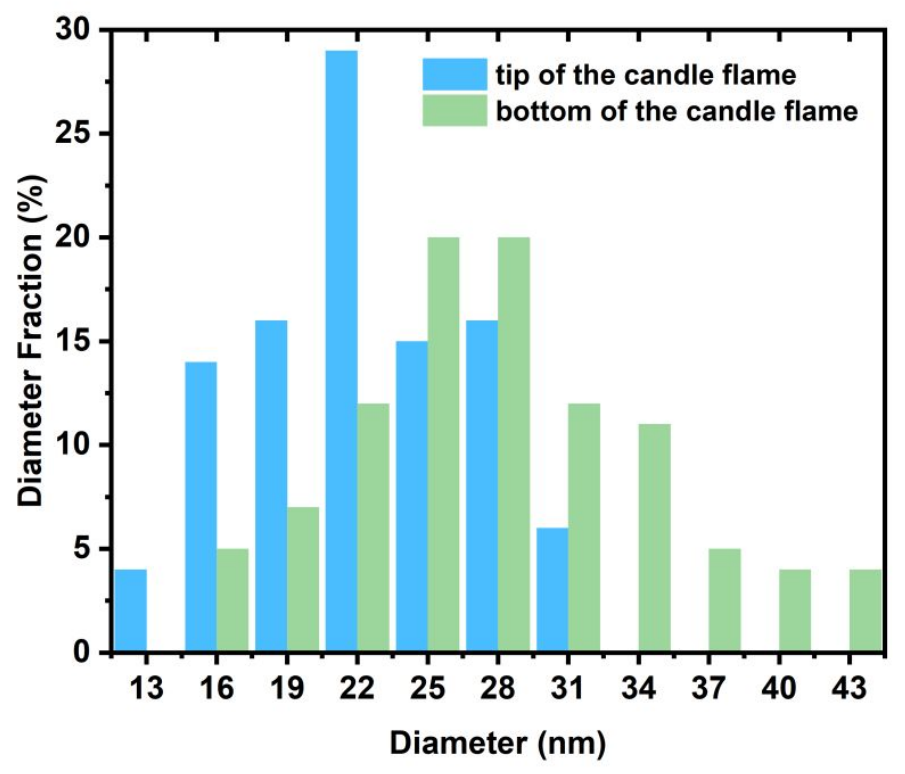

Figure S1 The size distributions of the CNPs gather from the tip of the flame and the bottom of the flame

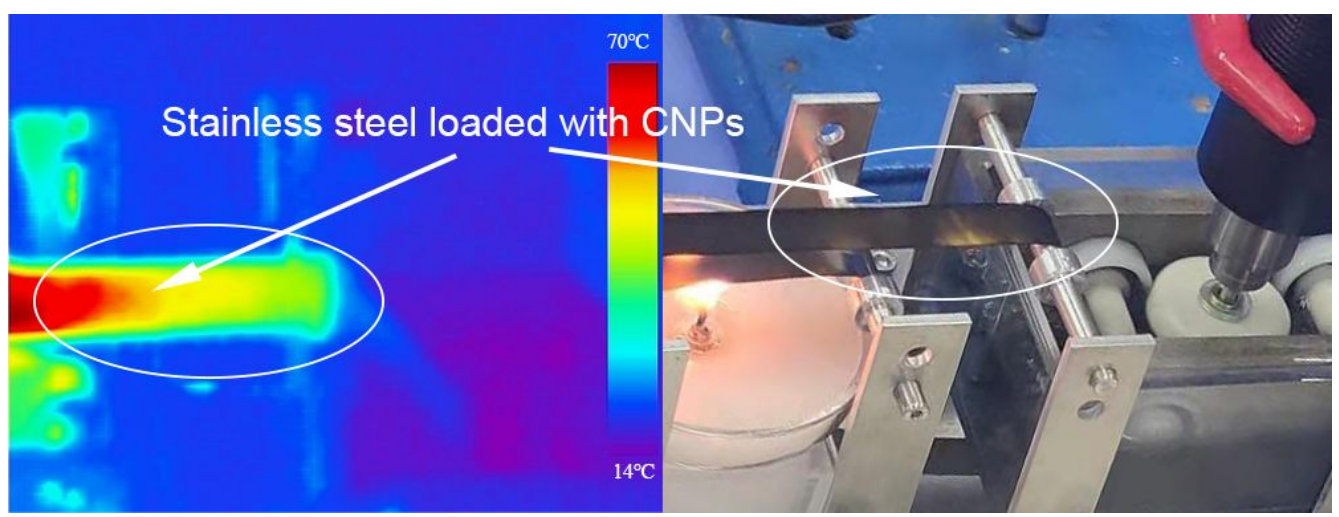

Figure S2 A infrared image and a photograph of the same area in the rotatingdeposition system. The infrared image shows that the stainless steel carrier rapidly cooled off after leaving the candle flame. 

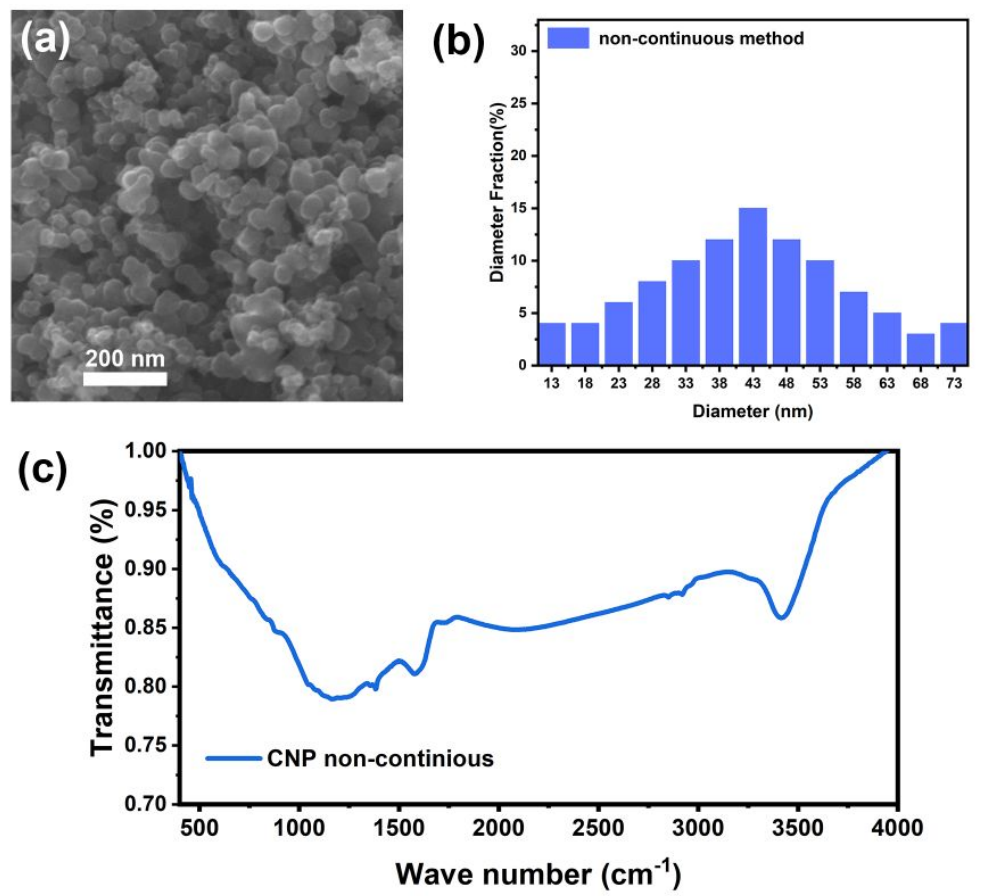

Figure S3 Morphology and Characteristics of CNPs by non-continuous method

a) SEM photograph of candle soot collected by non-continuous method.

These CNPs were collected by a piece of stainless steel above a candle. b) The size distributions of the CNPs collected by non-continuous method. Both SEM photograph and size distributions show that our obtained CNPs have a better uniformity than the CNPs by non-continuous method c) FTIR information of CNPs collected by non-continuous method. 

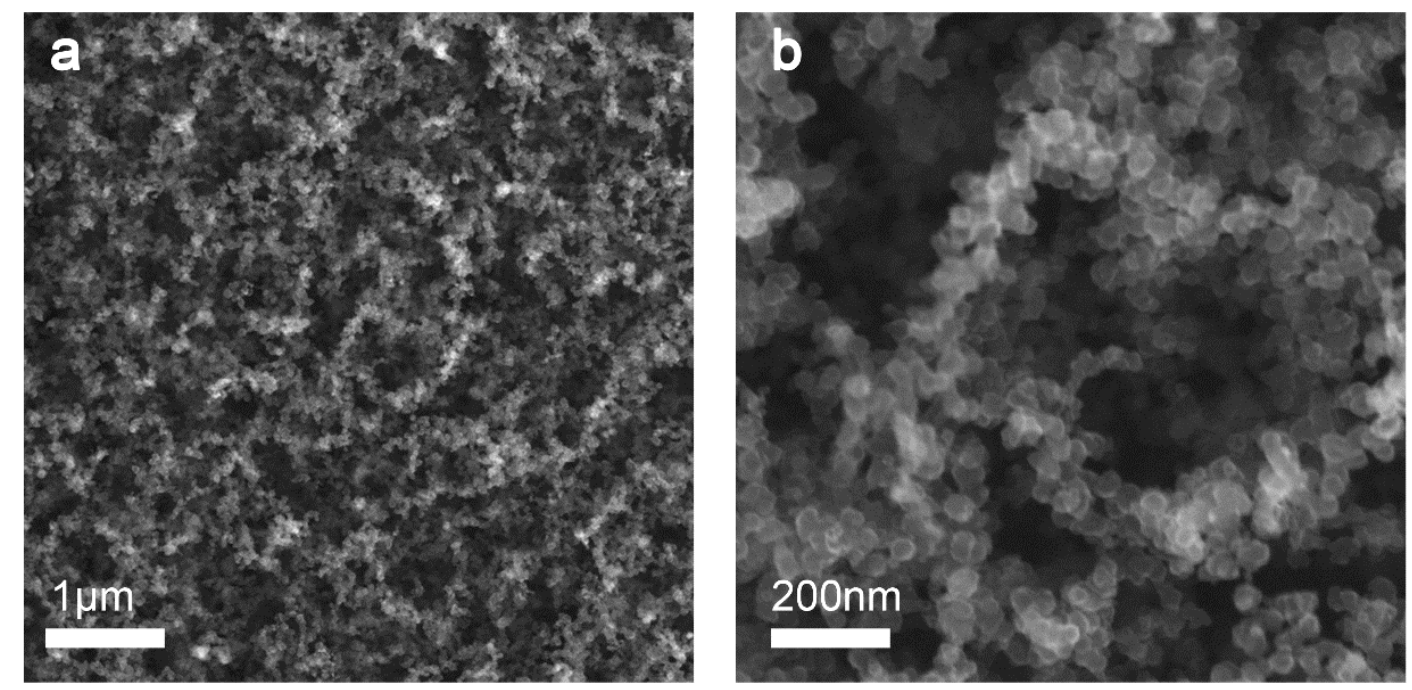

Figure S4 Candle soot directly deposited upon candle flame in different magnifications.

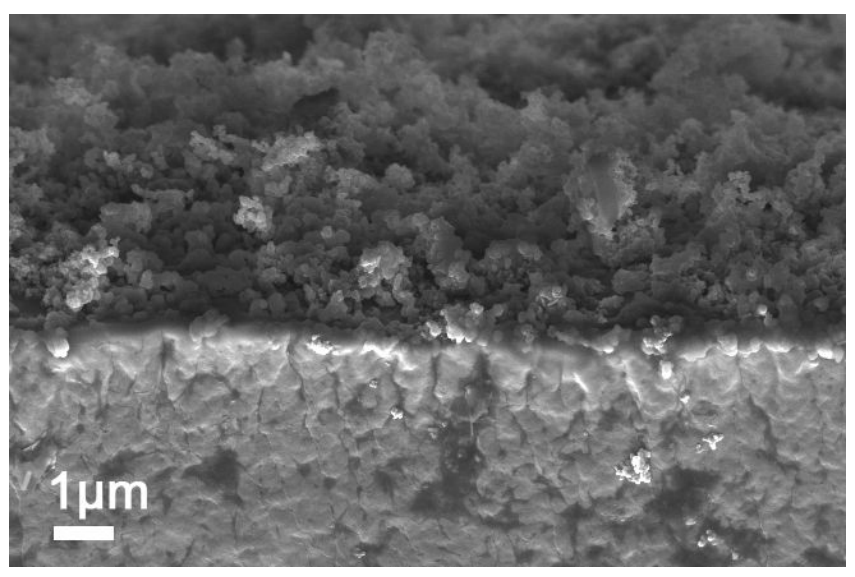

Figure S5 The SEM of the CPS section 


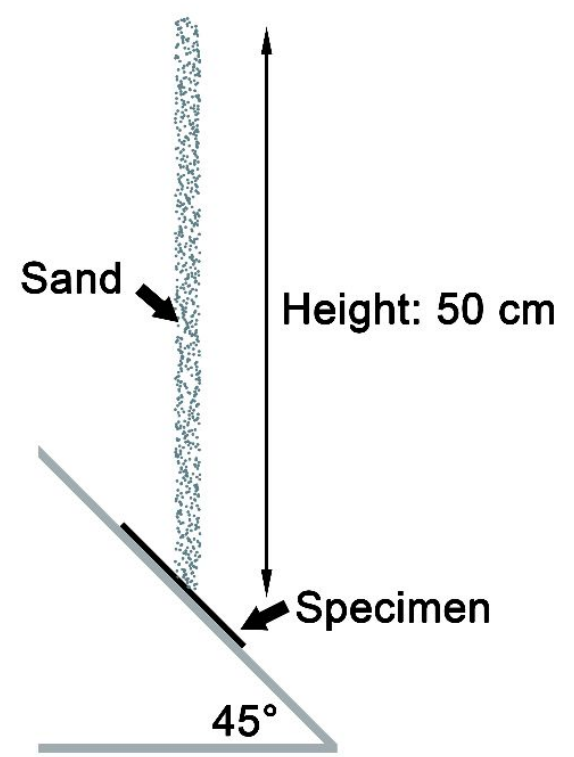

Figure S6 Abrasion test of CPS. CPS specimen were placed under a slope of $45^{\circ}$.

Sand struck the specimen from a height of $50 \mathrm{~cm}$.

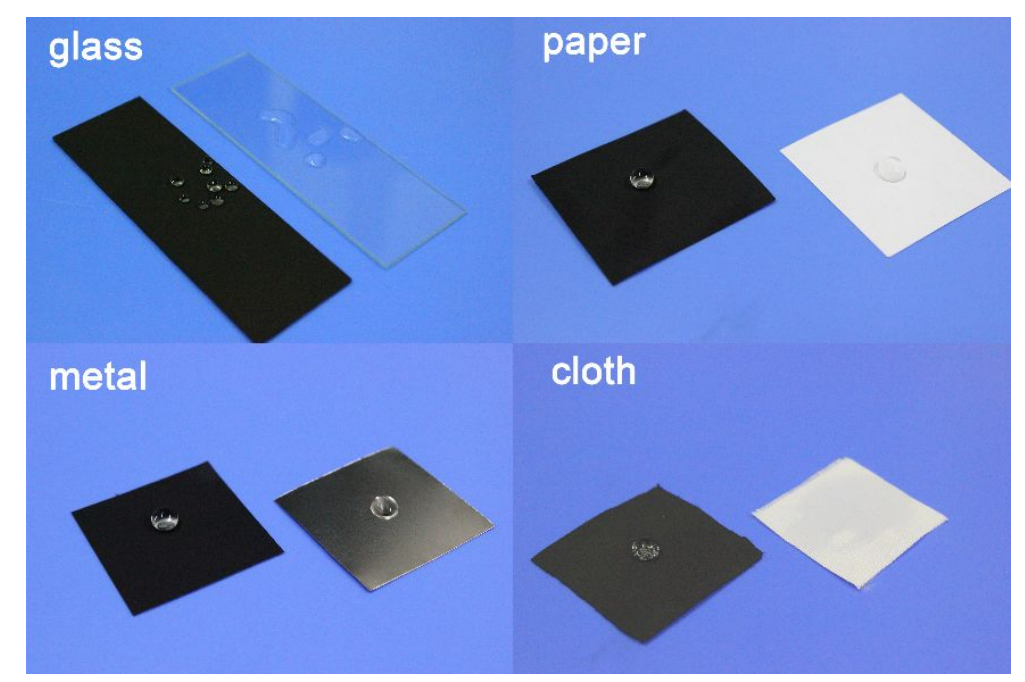

Figure S7 CPS coating on different types of substrates. 


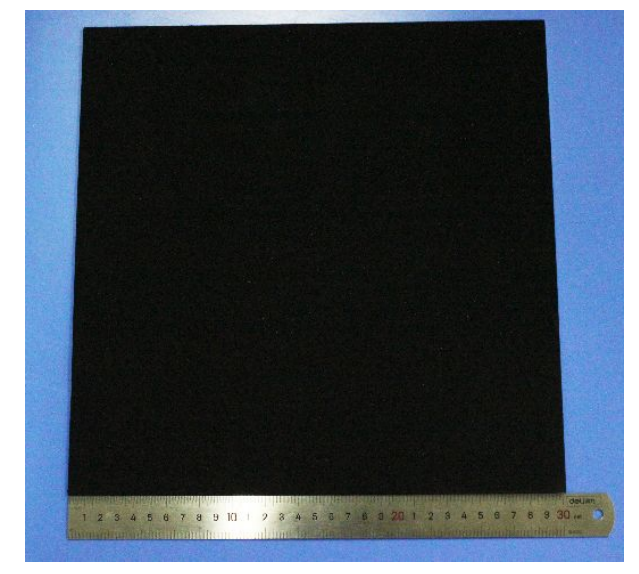

Figure S8 CPS coating on a $30 \mathrm{~cm}$ length, $30 \mathrm{~cm}$ width surface.

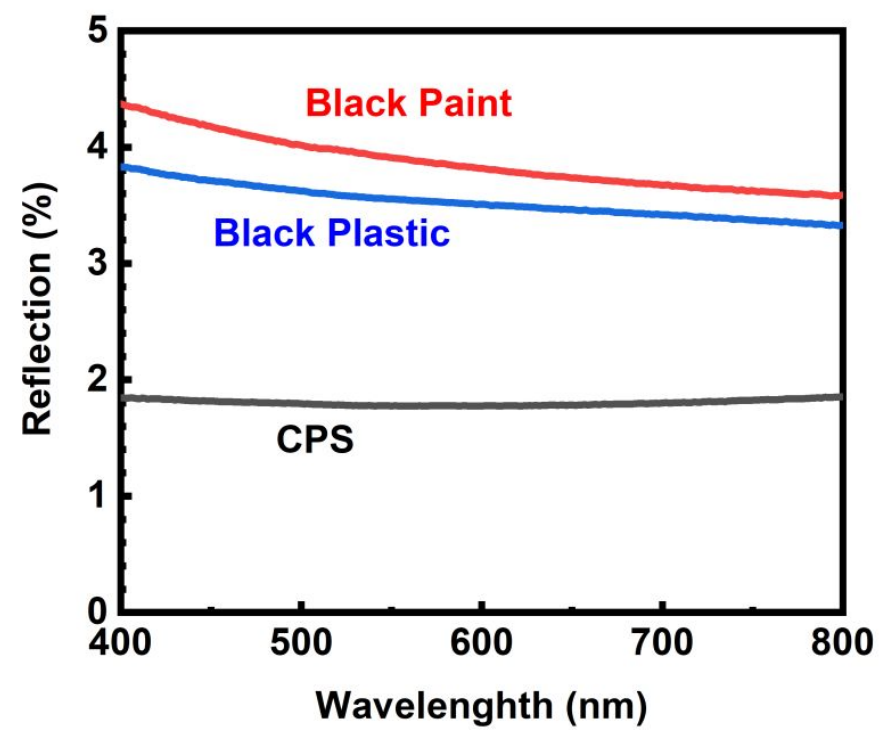

Figure S9 The reflection of visible light from different types of surfaces 


\section{Light source}

\section{Vedio camera}
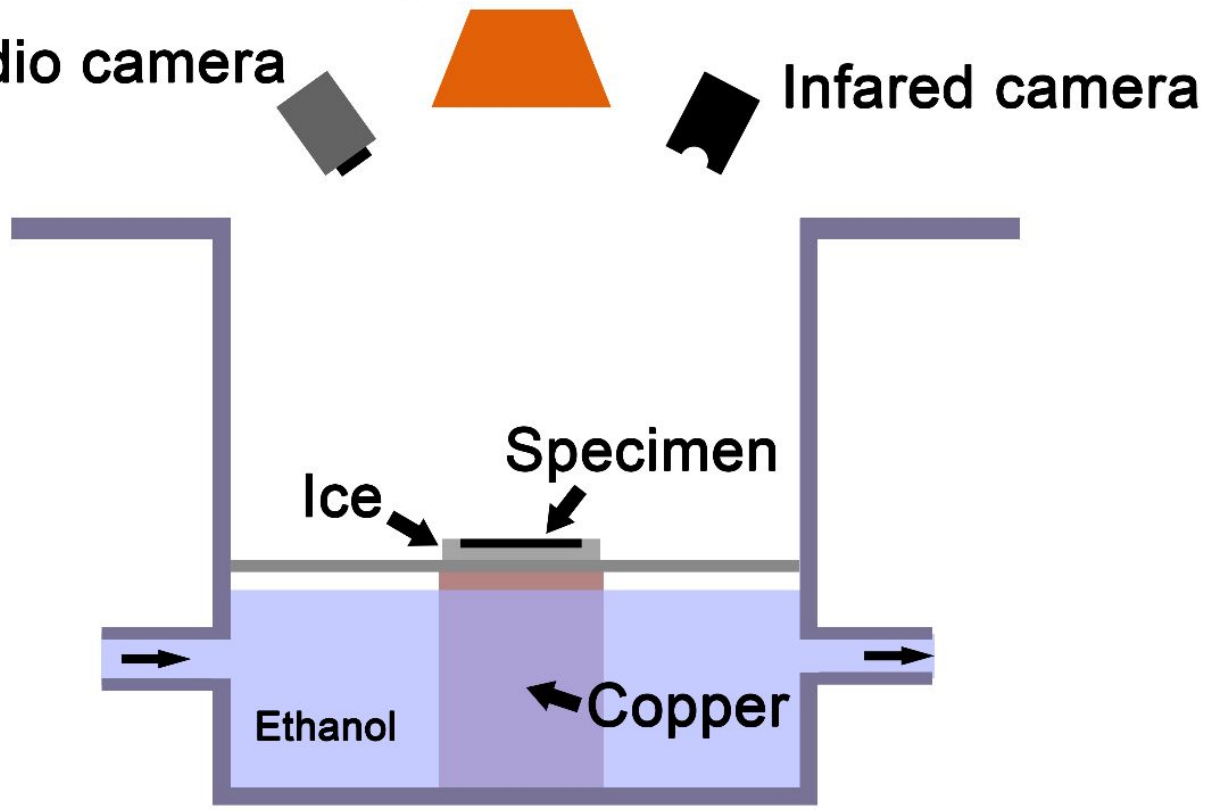

Figure S10 Testing system of ice-melting and frost melting. The ethanol was used as a cooling medium.

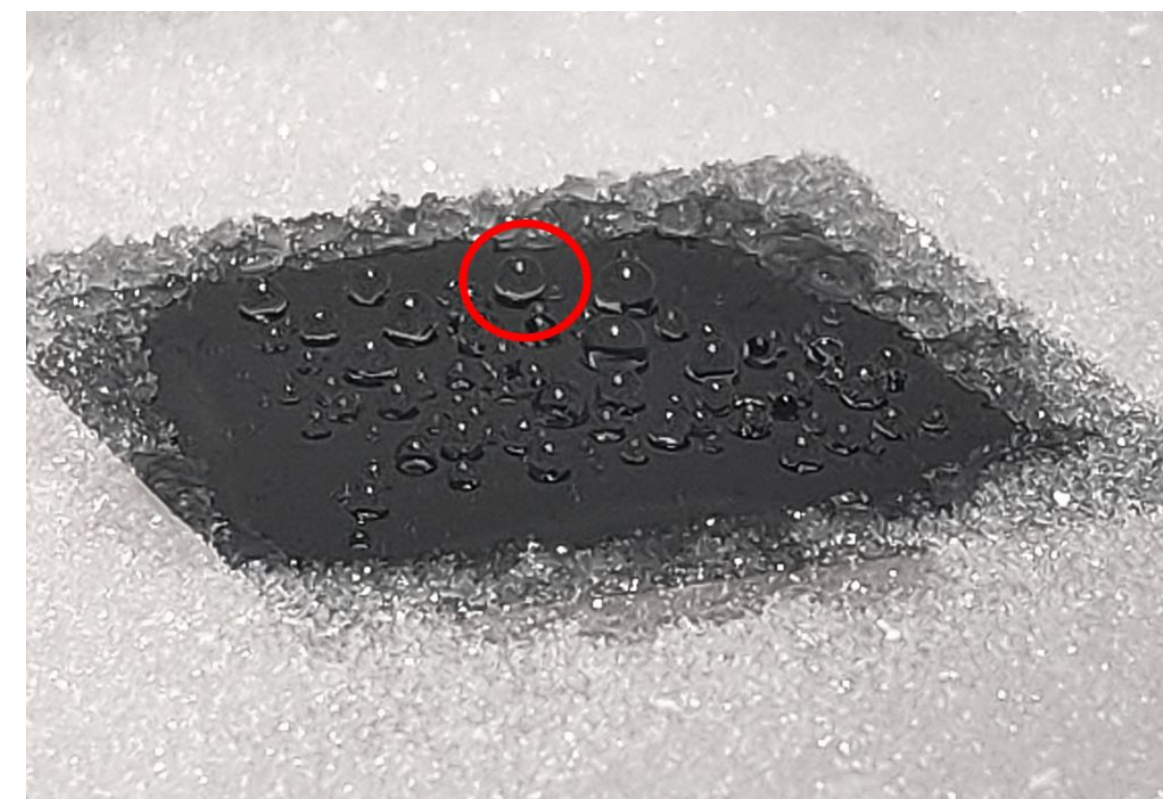

Figure S11 Melting water on CPS. The water drops showed that CPS surface remains superhydrophobic. 

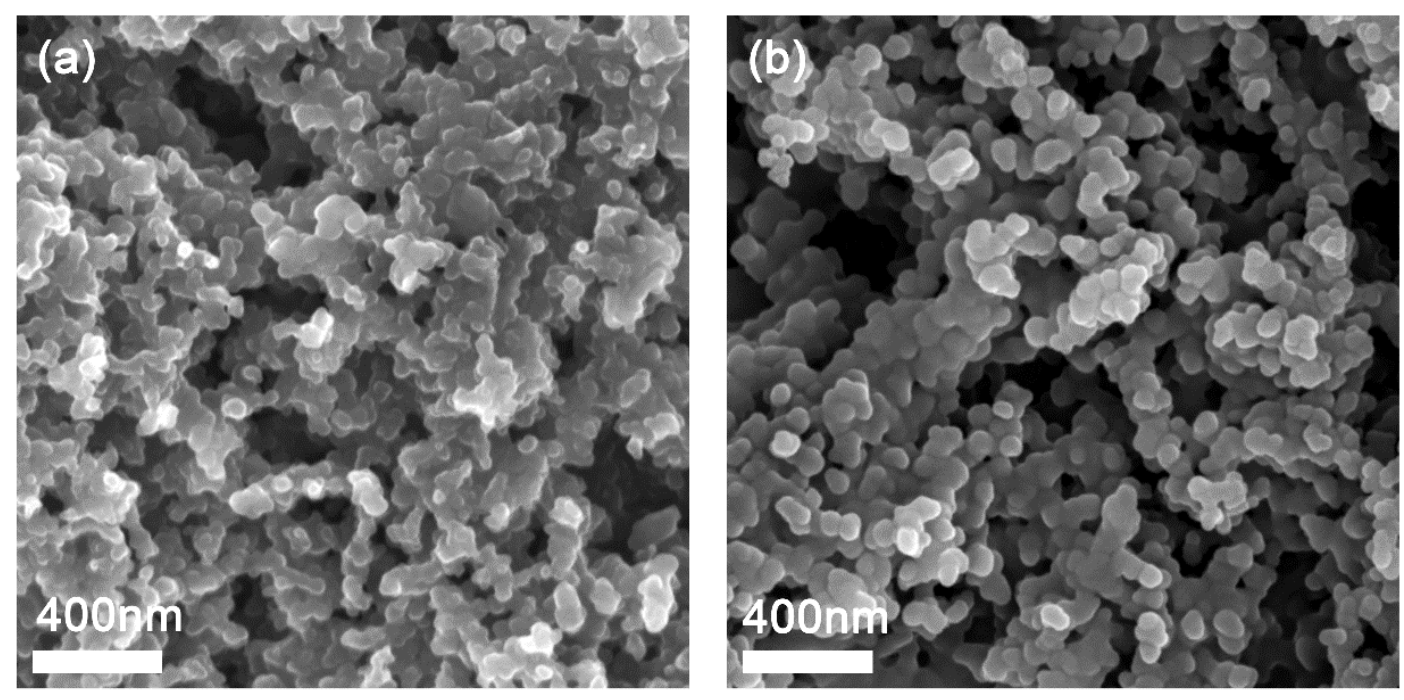

Figure S12 CPS before a) and after b) 20 circulations of icing-deicing. 